\title{
Zwitterionic polymer-based nanoparticles encapsulated with linalool for regulating central nervous system
}

Zhiguo Lu, ${ }^{\dagger,}$ Tianlu Zhang, ${ }^{\dagger}, \S$ Xiangyu Wang, ${ }^{\dagger}$ Jianze Wang, ${ }^{\dagger}$ Jie Shen, ${ }^{\dagger}$, Zuobing

Xiaol, * $\|, \wedge$ Lei Chen, ${ }^{*,+}$ and Xin Zhang ${ }^{*},+$

$\uparrow$ State Key Laboratory of Biochemical Engineering, Institute of Process Engineering,

Chinese Academy of Sciences, Beijing, 100190, PR China

\$Department of Obstetrics and Gynecology, Navy General Hospital of People

Liberation Army, Beijing 100048, PR China

$\S$ School of Chemical Engineering, University of Chinese Academy of Sciences,

Beijing, 100049, PR China

||Shanghai Research Institute of Fragrance and Flavor Industry, Shanghai 200232, PR

China

${ }^{\wedge}$ School of Perfume and Aroma Technology, Shanghai Institute of Technology,

Shanghai, 200233, PR China 


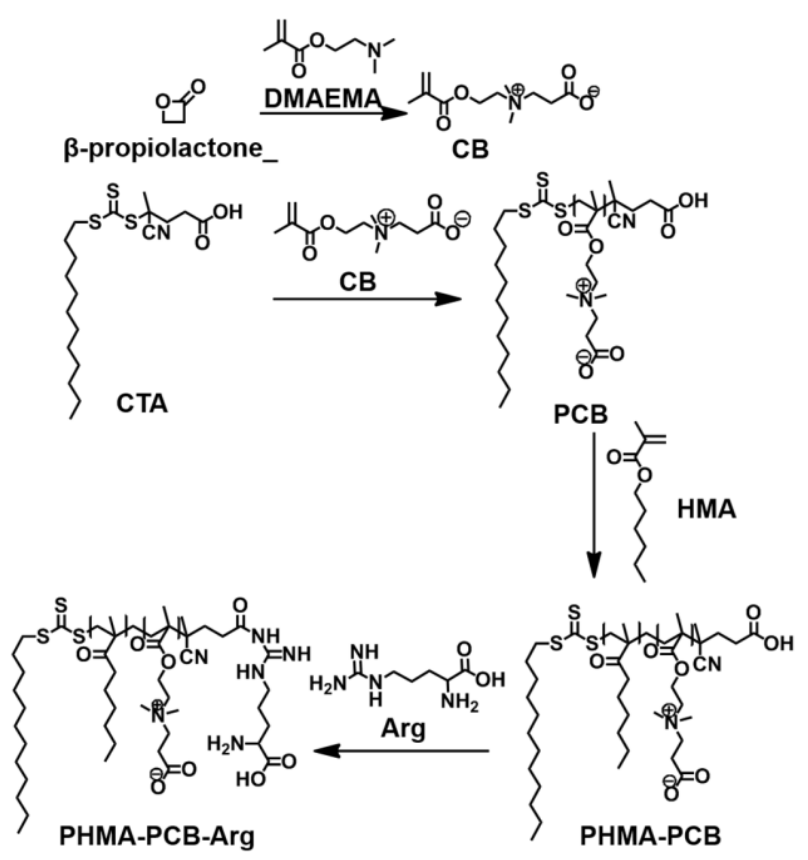

Figure S1. The synthetic route of PHMA-PCB-Arg.

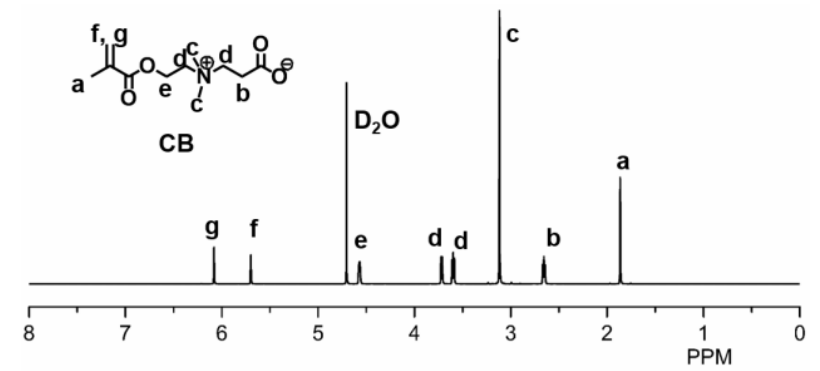

Figure S2. The ${ }^{1} \mathrm{H}$ NMR spectrum of CB.

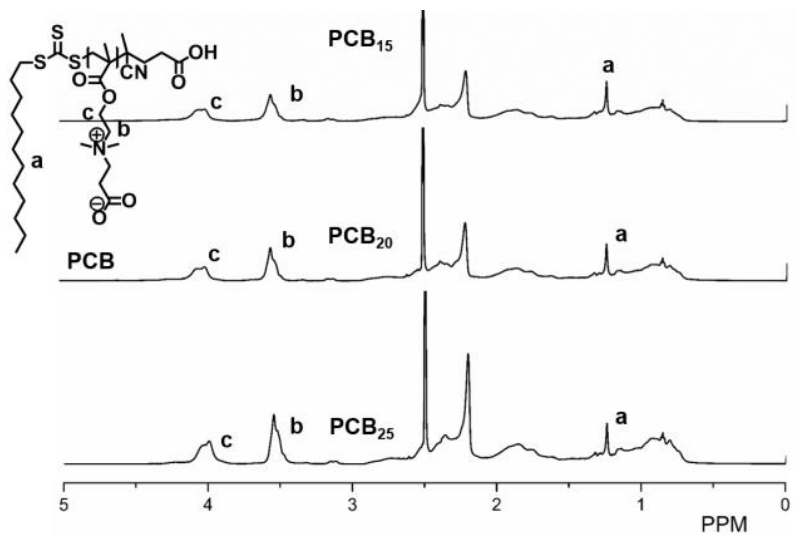

Figure $\mathrm{S} 3$. The ${ }^{1} \mathrm{H}$ NMR spectra of PCB. 


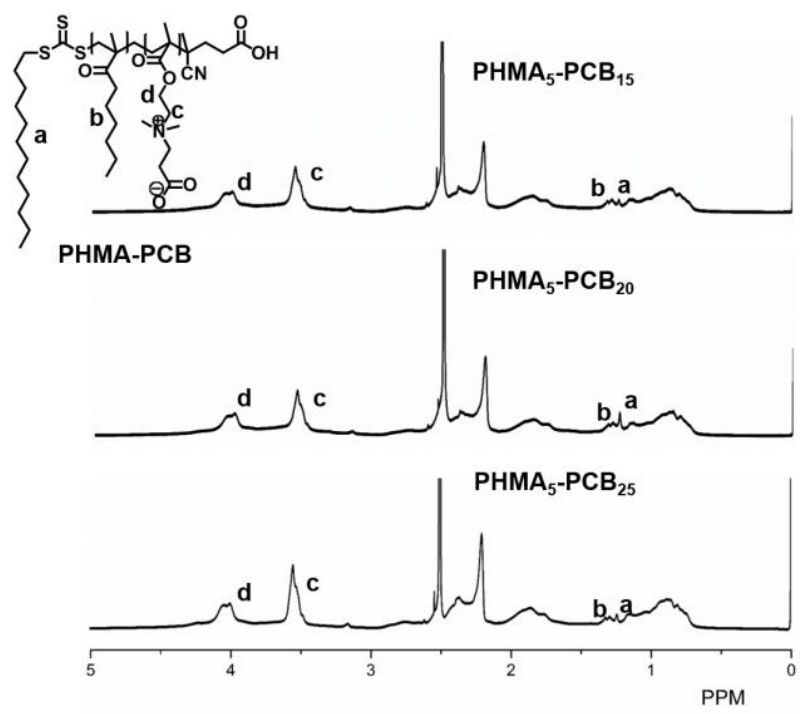

Figure S4. The ${ }^{1} \mathrm{H}$ NMR spectra of PHMA-PCB. 